\title{
Cadre juridique des allégations nutritionnelles et des allégations de santé
}

\author{
Guillaume COUSYN \\ Service Nutrition \& Diététique - Bureau D3. \\ Direction Générale de la Concurrence, \\ de la Consommation et de la Répression \\ des Fraudes 59, boulevard Vincent Auriol, \\ 75013 Paris \\ < guillaume.cousyn@dgccrf.finances.gouv.fr>
}

\begin{abstract}
Résumé : La santé constituant actuellement une des préoccupations majeures des consommateurs dans le domaine alimentaire, les industriels de l'agroalimentaire tentent de valoriser au mieux les caractéristiques nutritionnelles de leurs produits. Cette valorisation est pour l'instant principalement soumise à la responsabilité de ces industriels qui sont tenus de ne pas induire le consommateur en erreur. Le contrôle incombe à la Direction générale de la concurrence, de la consommation et de la répression des fraudes (DGCCRF) qui, selon les cas, peut interroger l'Agence française de sécurité sanitaire des aliments (Afssa) sur le fondement scientifique du message allégué. Toutefois, l'évolution alarmante de certains marqueurs de santé conduit les autorités compétentes à agir sur les différents facteurs susceptibles d'être impliqués dans cette évolution, dont la publicité. De nouvelles règles sont donc élaborées à Bruxelles, visant à améliorer le contrôle des allégations en leur soumettant notamment un régime d'autorisation préalable.
\end{abstract}

Mots-clés : étiquetage, publicité trompeuse, allégations nutritionnelles et de santé, DGCCRF, AFSSA, code de la consommation

gation nutritionnelle comme « toute représentation et tout message publicitaire qui énonce, suggère ou implique qu'une denrée alimentaire possède des propriétés nutritionnelles particulières:

- soit en raison de l'énergie (valeur calorique) qu'elle fournit ou ne fournit pas, ou qu'elle fournit à un taux réduit ou accru ;

- soit en raison des nutriments qu'elle contient ou ne contient pas ou qu'elle contient en proportion réduite ou accrue. » sant porter la responsabilité des produits à leur fabricant. Ainsi, il appartient aux industriels de vérifier que leurs produits sont sûrs pour le consommateur, qu'ils sont conformes aux dispositions en vigueur et que la publicité n'induit pas le consommateur en erreur. Cette dernière obligation, dite de "publicité non trompeuse », constitue la base du droit en matière de publicité.

\section{Allégations nutritionnelles et de santé : les dispositions en vigueur}

Les allégations constituent des mentions valorisantes appartenant au domaine de l'étiquetage facultatif, par opposition aux mentions obligatoires (dénomination de vente, date de péremption, numéro de lot...). Le caractère facultatif n'implique pas pour autant l'exemption de norme.

\section{Définitions}

À l'heure actuelle, seules les allégations nutritionnelles sont définies d'un point de vue réglementaire. L'article 4 du décret modifié $n^{\circ}$ 93-1130 du 27 septembre 1993 définit l'allé-
Concrètement, cette définition regroupe toutes les mentions quantitatives du type « source de... », «pauvre en... » ou encore «à teneur réduite en...", par exemple, accompagnées d'un nom de nutriment. II convient de noter que ce texte, qui transpose une directive ${ }^{1}$, dispose que ne peuvent être mentionnées que les allégations nutritionnelles concernant la valeur énergétique ainsi que les nutriments listés dans le texte (protéines, glucides, lipides, fibres, vita-

Les mentions dépassant la simple mention quantitative et faisant état d'un lien entre le produit ou une substance contenue dans le produit et la santé, ne sont pas encore définies réglementairement. Elles ne rentrent pas dans le champ d'application du décret précité.

${ }^{1}$ La directive $n^{\circ}$ 90/496 du 24 septembre 1990 du Conseil des communautés européennes relative au rapprochement des législations entre États membres concernant l'étiquetage nutritionnel des denrées alimentaires. mines et minéraux) et leurs composants.

\section{Dispositions en vigueur : deux interdictions majeures}

Toute mention valorisante relative à un produit, qu'elle soit définie par un texte réglementaire ou non, doit répondre à l'obligation générale de publicité non trompeuse, énoncée par I'article L. 121-1 du Code de la consommation. En résumé, il est interdit d'induire en erreur le consommateur par la publicité. Le délit de publicité trompeuse, qui a fait l'objet de nombreuses interprétations par les tribunaux, s'intéresse non seulement à la véracité du message qui est avancé mais également à la perception qu'en a le consommateur. Ce point est particulièrement important dans un domaine aussi complexe que celui de la nutrition.

Une deuxième règle $s$ 'applique, cette fois plus spécifiquement aux aliments : I'article R. 112-7 du Code de la consommation interdit, pour une denrée alimentaire, de faire état de propriétés de prévention, de traitement ou de guérison de maladies humaines.

En d'autres termes, les industriels sont libres d'alléguer sur leurs denrées toute propriété en lien avec la nutrition et la santé, sous réserve de s'assurer de la véracité de ces allégations (et donc de s'appuyer sur des données scientifiques valides) et de ne pas assimiler la denrée à un médicament.

\section{L'étiquetage nutritionnel}

Le décret $n^{\circ}$ 93-1130 soumet les allégations nutritionnelles à un ensemble de règles qui s'ajoutent aux règles précédemment décrites. En particulier, un étiquetage nutritionnel d'application facultative devient obligatoire dès lors qu'une allégation nutritionnelle figure 


\begin{tabular}{|c|c|c|}
\hline Allégations nutritionnelles sur... & \multicolumn{2}{|c|}{ Obligations d'étiquetage } \\
\hline $\begin{array}{l}\text { Valeur énergétique, quantité de protéines, de glucides } \\
\text { ou de lipides }\end{array}$ & \multicolumn{2}{|c|}{ Groupe 1 ou groupe 2 au choix } \\
\hline Sucres, acides gras saturés, fibres ou sodium & \multicolumn{2}{|l|}{ Groupe 2} \\
\hline $\begin{array}{l}\text { Acides gras mono-insaturés, polyinsaturés ou } \\
\text { cholestérol }\end{array}$ & $\begin{array}{c}\text { Groupe } 1+\text { acides gras } \\
\text { saturés } \\
\text { Ou } \\
\text { Groupe } 2\end{array}$ & + Élément \\
\hline $\begin{array}{l}\text { Amidon, polyols, vitamines et minéraux et autres } \\
\text { composants des nutriments listés }\end{array}$ & $\begin{array}{c}\text { Groupe } 1 \\
\text { Ou } \\
\text { Groupe } 2\end{array}$ & + Élément \\
\hline
\end{tabular}

sur l'étiquetage d'une denrée alimentaire (la définition de l'étiquetage incluant la publicité). Cet étiquetage présente une nature variable selon le(s) nutriment(s) faisant l'objet de l'allégation: un groupe 1 simplifié rassemble la valeur énergétique alors que le groupe 2, plus complet, y ajoute les sucres, les acides gras saturés, les fibres et le sodium (tableau 1).

Cet étiquetage doit permettre d'informer le consommateur sur les différentes caractéristiques nutritionnelles de la denrée et ainsi lui permettre de mieux éclairer son choix, sans se laisser guider uniquement par les mentions valorisantes.

\section{Allégations nutritionnelles et de santé : la procédure de contrôle}

La DGCCRF est I'administration chargée de contrôler la bonne application des articles du Code de la consommation relatifs à la publicité trompeuse. L'article L. 121-2 habilite les agents de la DGCCRF à exiger d'un annonceur I'ensemble des documents de nature à justifier le fondement de son allégation. Cette procédure vise à protéger la santé et les intérêts des consommateurs et à garantir une concurrence loyale entre les différents acteurs.

\section{La saisine de I'Afssa}

Dans un domaine aussi technique que celui de la nutrition, le gestionnaire du risque, en I'occurrence la DGCCRF, peut être amené à saisir l'instance d'évaluation, l'Afssa. Celle-ci sera alors chargée d'évaluer les documents fournis par l'industriel (bibliographie, études cliniques...) afin de rendre un avis sur le fondement scientifique de l'allégation. Au regard de I'avis, il appartient alors au gestionnaire de décider s'il considère la publicité comme trompeuse ou non, sous réserve, bien entendu, de l'appréciation souveraine des tribunaux.

\section{Les données scientifiques disponibles}

Afin de vérifier la véracité du message affiché, la DGCCRF dispose également de plusieurs avis scientifiques. En premier lieu, l'avis de la Cedap du 8 juillet 1998 fixe en effet, pour plusieurs substances, les seuils à partir desquels les différents niveaux d'allégation nutritionnelle (source, riche, pauvre, exempt...) sont significatifs. Un autre avis de la Cedap, daté du 18 décembre 1996, donne une liste d'allégations faisant le lien entre des nutriments et des fonctions de l'organisme reconnues comme avérées.

En outre, l'Afssa, à l'occasion d'avis relatifs à des produits ou de groupes de travail, a établi des certains messages. À titre d'exemple, le groupe de travail sur les acides gras de la famille des oméga 3 a indiqué les critères à respecter pour alléguer sur le rôle des oméga 3 dans le bon fonctionnement cardiovasculaire.

Ce corpus de documents permet de simplifier la procédure et de ne pas saisir l'Afssa systématiquement.

\section{Recommandations}

Les industriels sont tenus d'indiquer sur leurs étiquetages des messages qui sont vrais scientifiquement tout en étant compréhensibles par le consommateur, ce qui peut constituer dans certains cas une réelle gageure.

La justification des allégations nécessite d'étudier de façon approfondie la bibliographie existante, c'est-à-dire de rassembler les études venant à l'appui de l'effet allégué mais également celles qui pourraient le contredire. En outre, selon le niveau d'allégation, il pourra être demandé à l'industriel de réaliser des études cliniques significatives permettant de vérifier la véracité du message. Ces études, très coûteuses, constituent le socle d'un dossier justificatif complété par la bibliographie et les études disponibles (in vitro et sur l'animal). critères permettant de vérifier la véracité de
De nombreux guides ont été réalisés ou sont en cours d'élaboration afin d'aider les industriels dans la réalisation d'allégations et des justificatifs permettant leur emploi. Ainsi des guides de bonnes pratiques ont été diffusés par l'Association nationale des industries agroalimentaires (Ania), par la Confederation of the food and drink industries of the EU (CIAA), le Bureau de la vérification de la publicité (BVP)... De son côté, l'Afssa a émis des lignes directrices à la réalisation de dossiers. Enfin, I'International Life Sciences Institute (ILSI) dans le cadre de son projet Passclaim doit diffuser un document consensuel facilitant l'évaluation des preuves venant à l'appui des allégations, ce qui impliquera notamment de définir les marqueurs biologiques pertinents.

\section{Les évolutions réglementaires discutées au niveau européen}

Afin de garantir la loyauté des transactions et permettre la libre circulation des marchandises, la Commission a proposé un projet de règlement visant à encadrer par des dispositions spécifiques les allégations nutritionnelles et de santé.

Le projet de texte, tel qu'il est rédigé actuellement, propose :

- une définition pour les différents types d'allégations ;

- des listes d'allégations autorisées avec les éventuels critères à respecter ;

- des procédures d'autorisation afin de compléter les listes ;

- des conditions restrictives à l'emploi d'allégations.

Les principales innovations par rapport au système actuel résident $d^{\prime}$ une part dans la mise en place d'une autorisation préalable pour ces allégations et la publication des listes d'allégations autorisées. Cela permettra notamment aux consommateurs de connaître ce qui est avéré scientifiquement. D'autre part, il a été proposé que seules les denrées dont le profil nutritionnel (sans que celui-ci n'ait encore été défini) puissent faire état de leurs propriétés nutritionnelles. La France a obtenu que soit prise en compte, dans la définition de ces profils, la place de l'aliment dans le régime alimentaire. II paraît en effet légitime qu'une huile, bien que composée quasi uniquement de lipides, puisse mettre en avant l'intérêt de ses acides gras si ceux-ci présentent des bénéfices pour la santé.

Enfin, le texte prévoit des interdictions (par exemple, l'interdiction d'allégations sur les boissons alcoolisées). 
Le système de contrôle actuel présente plusieurs inconvénients. Le principal réside dans la subjectivité de l'appréciation du caractère non trompeur de l'allégation. Par ailleurs, le consommateur ne peut à aucun moment faire la distinction entre les allégations avérées et les publicités basées sur des données scientifiques insuffisantes. En outre, les services de contrôle peuvent difficilement vérifier la totalité des messages présents sur le marché. Une telle situation peut provoquer une concurrence déloyale. C'est pourquoi la Commission euro- péenne, motivée par les résolutions du Conseil de l'Union européenne, a proposé des règles visant à restreindre considérablement la liberté accordée, jusqu'à présent, dans ce domaine aux industriels. 